MR. MIGUEL LÓPEZ FRAILE LÓPEZ (Orcid ID : 0000-0001-6656-5387)

PROF. SHIV KUMAR SARIN (Orcid ID : 0000-0002-0544-5610)

DR. JOAN GENESCA (Orcid ID : 0000-0002-0831-8422)

DR. JUAN CARLOS GARCIA-PAGAN (Orcid ID : 0000-0001-9032-4954)

Article type : Original

Title: Congenital extrahepatic portosystemic shunts (Abernethy malformation):

An international observational study

\title{
Authors list:
}

Baiges Anna ${ }^{1}$, Turon Fanny ${ }^{1}$, Simón-Talero Macarena ${ }^{2}$, Tasayco Stephanie ${ }^{2}$, Bueno Javier $^{3}$, Zekrini Kamal $^{4}$, Plessier Aurélie ${ }^{4}$, Franchi-Abella Stéphanie ${ }^{5}$, Guerin Florent ${ }^{5}$, Mukund Amar ${ }^{6}$, Eapen $\mathrm{CE}^{7}$, Goel Ashish ${ }^{7}$, Shyamkumar Nidugala Keshava ${ }^{8}$, Coenen Sandra ${ }^{9}$, De Gottardi Andrea ${ }^{10}$, Majumdar Avik $^{11}$, Onali Simona ${ }^{11}$, Shukla Akash ${ }^{12}$, Carrilho Flair José ${ }^{13}$, Nacif Lucas ${ }^{13}$, Primignani Massimo ${ }^{14}$, Tosetti Giulia $^{14}$, La Mura Vicenzo ${ }^{14}$, Nevens Frederik ${ }^{15}$, Witters Peter ${ }^{15}$, Tripathi Dhiraj ${ }^{16}$, Tellez Luis ${ }^{17}$, Martínez Javier ${ }^{17}$, Álvarez-Navascués Carmen ${ }^{18}$, Fraile Miguel ${ }^{18}$, Procopet Bogdan ${ }^{19}$, Piscaglia Fabio $^{20}$, de Koning Barbara ${ }^{21}$, Llop Elba ${ }^{22}$, Cristobal Mario Romero ${ }^{23}$, Tjwa Eric ${ }^{24}$, Monescillo-Francia Alberto $^{25}$, Senzolo Marco ${ }^{26}$, Perez-LaFuente Mercedes ${ }^{27}$, Segarra Antonio ${ }^{27}$, Sarin Shiv Kumar ${ }^{28}$, Hernández-Gea Virginia ${ }^{1}$, Patch David ${ }^{11}{\text { Laleman } \text { Wim }^{15} \text {, Hartog Hermien }}^{9}$, Valla Dominique ${ }^{4}$, Genescà Joan², García-Pagán Joan Carles ${ }^{1}$ for the REHEVASC, VALDIG an EASL consortium and Abernethy group.

This article has been accepted for publication and undergone full peer review but has not been through the copyediting, typesetting, pagination and proofreading process, which may lead to differences between this version and the Version of Record. Please cite this article as doi: 10.1002/hep.30817

This article is protected by copyright. All rights reserved. 
1. Barcelona Hepatic Hemodynamic Laboratory, Liver Unit, Hospital Clínic de Barcelona, IDIBAPS, CIBERehd, Universitat de Barcelona, Spain

2. Liver Unit, Department of Internal Medicine, Hospital Universitari Vall d'Hebrón, VHIR, CIBERehd, Universitat Autònoma de Barcelona, Spain

3. Pediatric Surgery Department, Hospital Universitari Vall d'Hebrón. Universitat Autònoma de Barcelona, Spain

4. DHU Unity, Pôle des Maladies de l'Appareil Digestif, Service d'Hépatologie, Centre de Référence des Maladies Vasculaires du Foie, Hôpital Beaujon, AP-HP, Clichy, France

5. Service d'Hépatologie et de Transplantation Hépatique et de radiologie Pédiatriques. Groupement Hospitalier Paris Sud (GHUPS), Hôpital Bicêtre, LE KREMLIN BICETRE, France

6. Department of Radiology, Institute of Liver and Biliary Sciences, Vasant Kunj, New Delhi, India

7. Hepatology Department, Christian Medical College, Vellore, India

8. Department of Radiology, Christian Medical College, Vellore, India

9. Department of Gastroenterology and Hepatology, Erasmus Medical Center, Rotterdam, the Netherlands.

10. Hepatology, Clinic of Visceral Surgery and Medicine, Inselspital and Department of Clinical Research, University of Bern, Switzerland

11. Sheila Sherlock Liver Unit and UCL Institute for Liver and Digestive Health, Royal Free Hospital and UCL, London, United Kingdom

12. Department of Gastroenterology, Seth G S Medical College \& KEM Hospital, Mumbai, Maharashtra, India

13. Digestive Organ Transplantation Division, Department of Gastroenterology, Faculty of Medicine, University of São Paulo, SP, Brazil.

14. A. M. e A. Migliavacca" Center for Liver Disease, Division of Gastroenterology and Hepatology, Fondazione IRCCS Ca' Granda Ospedale Maggiore Policlinico, University of Milan, Milan, Italy

15. Department of Gastroenterology and Hepatology, University Hospitals Leuven, KU Leuven, Leuven, Belgium

16. Liver Unit, Queen Elisabeth Hospital, Birmingham, UK

17. Department of Gastroenterology and Hepatology, Hospital Universitario Ramón y Cajal, IRYCIS, CIBERedh, University of Alcalá, Madrid, Spain

This article is protected by copyright. All rights reserved. 
18. Aparato Digestivo, Hospital Universitario Central de Asturias HUCA, España

19. Department of Gastroenterology, $3^{\text {rd }}$ Medical Clinic, University of Medicine and Pharmacy "Iuliu Hatieganu". Regional Institute of Gastroenterology and Hepatology "O Fodor", Cluj-Napoca, Romania 20. Unit of Internal Medicine, Department of Medical and Surgical Sciences, University of Bologna, S. Orsola-Malpighi Hospital, Bologna, Italy.

21. Division of Pediatric Gastroenterology, Erasmus MC-Sophia Children's Hospital, Rotterdam, The Netherlands.

22. Liver Unit, Hospital U. Puerta de Hierro, Universidad Autónoma de Madrid, Madrid, Spain.

23. Digestive Diseases and Centro de Investigación Biomédica en Red de Enfermedades Hepáticas y Digestivas Hospital General Universitario Gregorio Marañón Instituto de Investigación Sanitaria Gregorio Marañón Facultad de Medicina, Universidad Complutense, Madrid, Spain

24. Department of Gastroenterology and Hepatology, Radboud University Medical Centre, Nijmegen, The Netherlands

25. Digestivo, Complejo Hospitalario Universitario Insular-Materno Infantil, España

26. Multivisceral Transplant Unit, Department of Surgery, Oncology and Gastroenterology, Padua University Hospital, Padua, Italy

27. Interventional Radiology Unit, Hospital Universitari Vall d'Hebrón. Universitat Autònoma de Barcelona, Spain.

28. Department of Hepatology, Institute of Liver and Biliary Sciences, New Delhi, India.

Abernethy Group: García-Criado Ángeles, Darnell Anna, Belmonte Ernest, Ferrusquía-Acosta José, Magaz Marta, Vidal-González Judit, Horia Stefanescu, Oana Nicoara-Farcau, Joseph Philip, Zachariah Uday, Moses Vinu, Mammen Suraj, Ahmed Munawwar, Koshy George, Eapen Anu, Sajith K.G., Vyas F.L., Raju R.S., Rymbai Manbha L., Arulkumar S., Ramachandran Jeyamani, Elias Elwyn

This article is protected by copyright. All rights reserved. 


\section{Footnote Page}

Keywords: Congenital malformation, extrahepatic portosytemic shunt, Abernethy

\section{Contact information for corresponding author:}

Juan Carlos Garcia-Pagan, MD, PhD, Barcelona Hepatic Hemodynamic Laboratory, Liver Unit, Hospital Clinic, Villarroel 170, Barcelona 08036, Spain. Fax: +34 932279856; e-mail address: jcgarcia@clinic.cat

List of abbreviations in alphabetical order: CEPS (congenital Extrahepatic Portosystemic Shunt), CT (Computed Tomography), FU (follow up), GP (Globus Pallidum), HCC (HepatoCellular Carcinoma), HE (Hepatic Encephalopathy), HPS (Hepatopulmonary Syndrome), IVC (Inferior Vena Cava), IPSS (Intrahepatic Portosystemic Shunt), MRI (Magnetic Resonance Imaging), OLT (Orthotopic Liver Transplantation), PaHT (Pulmonary arterial Hypertension), US (UltraSound), REHEVASC (Registro de Enfermedades Hepáticas Vasculares), VALDIG (Vascular Liver Disease Interest Group).

Electronic Number of figures and tables: 4 figures, 1 table, 2 supplementary figures, 3 supplementary tables

Financial support statement: This study was supported by the Ministry of Education and Science (SAF-2016-75767-R), and Instituto de Salud Carlos III (PIE15/00027), FEDER "Una manera de hacer Europa"). The Centro de Investigación Biomédica en Red de Enfermedades Hepáticas y Digestivas (CIBERehd) is funded by the Instituto de Salud Carlos III. IDIBAPS CERCA Programme/ Generalitat de Catalunya, Societat Catalana de Digestologia. REHEVASC is partially funded by Asociación Española para Estudio del Hígado (AEEH). VALDIG is supported by a grant from the European Association for the Study of the Liver (EASL).

Macarena Simón-Talero is a recipient of a Juan Rodés grant (JR17/00029) from Instituto de Salud Carlos III, Spain. Stephanie Tasayco is a recipient of a PFIS grant (Fl16/00238) from Instituto de Salud Carlos III, Spain. Joan Genescà is supported by Instituto de Salud Carlos III, Spain (Research

This article is protected by copyright. All rights reserved. 
Intensification grant, PI15/00066 and PI18/00947) and by European Union (ERDF/ESF, "Investing in your future").

Disclosures and conflict of interest statement: The authors who have taken part in this study declare that they do not have anything to disclose regarding funding or conflict of interest with respect to this manuscript. All authors participated meaningfully in the study and have seen and approved the final manuscript.

Authors contributions: Turon, Hernández-Gea and García Pagán designed the study. Baiges Anna, Turon Fanny, Simón-Talero Macarena, Tasayco Stephanie, Bueno Javier, Zekrini Kamal, Plessier Aurélie, Franchi-Abella Stéphanie, Guerin Florent, Mukund Amar, Eapen CE, Goel Ashish, Shyamkumar Nidugala Keshava, Coenen Sandra, De Gottardi Andrea, Majumdar Avik Onali Simona, Shukla Akash, Carrilho Flair José, Nacif Lucas, Primignani Massimo, Tosetti Giulia, La Mura Vicenzo, Nevens Frederik, Witters Peter, Tripathi Dhiraj, Tellez Luis, Martínez Javier, Álvarez-Navascués Carmen, Fraile Miguel, Procopet Bogdan, Piscaglia Fabio, de Koning Barbara, Llop Elba, Cristobal Mario Romero, Tjwa Eric, Monescillo-Francia Alberto, Senzolo Marco, Perez-La Fuente Mercedes, Segarra Antonio, Sarin Shiv Kumar, Hernández-Gea Virginia, Patch David, Laleman Wim, Hartog Hermien, Valla Dominique, Genescà Joan, García-Pagán Joan Carles collected and reviewed the data and reviewed the manuscript. Baiges Anna, Turon Fanny, Hernández-Gea Virginia and García Pagán Juan Carlos collected, reviewed and analyzed the data and wrote the manuscript.

\section{Abstract}

Background and Aims: Congenital extrahepatic portosystemic shunt (CEPS) or Abernethy malformation is a rare condition in which splanchnic venous blood bypasses the liver draining directly into systemic circulation through a congenital shunt. Patients may develop hepatic encephalopathy $(\mathrm{HE})$, pulmonary hypertension $(\mathrm{PaHT})$ or liver tumors, among other complications. However, the actual incidence of such complications is unknown mainly due to the lack of a protocolized approach to these patients. This study characterizes the clinical manifestations and outcome of a large cohort of CEPS patients with the aim of proposing a guide for their management.

This article is protected by copyright. All rights reserved. 
Methods: Observational multicenter international study.

Results: Sixty-six patients were included, median age at the end of follow-up was 30 years. Nineteen patients (28\%) presented HE. Ten, 20 and 30 year HE incidence rate were 13, 24 and 28\%, respectively. No clinical factors predicted $\mathrm{HE}$.

Twenty-five patients had benign nodular lesions. Ten patients developed adenomas (median age: 18 years) and another 8 developed HCC (median age: 39 years).

Out of 10 patients with dyspnea, PaHT was diagnosed in 8 and hepatopulmonary syndrome in 2 . Pulmonary complications were only screened for in 19 asymptomatic patients and PaHT was identified in 2.

Six patients underwent liver transplantation (LT) for HCC or adenoma. Shunt closure was performed in 15 patients with improvement/stability/cure of CEPS manifestations.

Conclusion: CEPS patients may develop severe complications. Screening for asymptomatic complications and close surveillance is needed. Shunt closure should be considered both as a therapeutic and prophylactic approach.

\section{Introduction}

Congenital extrahepatic portosystemic shunts (CEPS) are a rare condition in which most of the intestinal and splenic venous blood bypasses the portal vein and the liver, draining directly into systemic veins through abnormal communications. Anatomically, CEPS has been classified in two types (1)'(2). Type I CEPS is characterized by the absence of intrahepatic portal vein branches and an end-to-side portocaval shunt, while in type II CEPS the intrahepatic veins are hypoplastic but patent and a side-to side shunt diverts blood from the portal vein to the inferior vena cava. Type I CEPS can be further classified into type la, when the superior mesenteric and splenic vein drain separately into inferior cava veins (IVC), and type lb, when these veins form a common trunk before draining into the IVC.

Recently, more detailed anatomical subclassifications (3)'(4) have been described, correlating the anatomy of the shunt to the surgical approach required for its closure(4)'(5). It has also been stated that, to accurately assess the patency of intrahepatic veins, an angiography with occlusion of the shunt should be performed, to distinguish if the assumed absence of intrahepatic veins could in fact be concealing remnant hypoplastic hepatic branches (3)'(4)'(6).

This article is protected by copyright. All rights reserved. 
CEPS is usually associated with congenital anomalies, the most frequent being cardiac abnormalities, genitourinary malformations, and musculoskeletal defects. Biliary atresia has also been associated to CEPS'(2)'(7) (8)'(9).

In countries routinely performing neonatal screening for hereditary galactosemia, it has been estimated that the incidence of congenital portosystemic shunts is around 1 in 30,000 births (10)'(11) (high levels of galactose can be found in newborns with congenital portosystemic shunts because galactose bypasses the liver). However, the usefulness of galactosemia screening to infer CEPS prevalence is debatable because other circumstances could cause false positive results, galactosemia levels cannot differentiate intra and extrahepatic shunts and, finally, it may underestimate its real prevalence since not all patients with CEPS harbor hypergalactosemia.

To our knowledge, less than 300 cases of CEPS have been reported in the literature. The spectrum of clinical variants of CEPS ranges from completely asymptomatic forms to severe forms of hepatic encephalopathy (HE)'(12)' '(13), hepatopulmonar syndrome (HPS) and pulmonary arterial hypertension (PaHT)(14-16).Nodular liver lesions are frequent in patients with CEPS and although most of these nodules are benign, hepatocellular carcinomas and adenomas, among other neoplastic lesions, have been reported (17)' '(18). However, the vast majority of these publications are single case reports providing only a cross-sectional description without follow-up. Although some series have been reported (3)'(4)'(19)'(20) most mix patients with CEPS and intrahepatic congenital portosystemic shunts (IPSS). The natural history of these two entities might be different, being IPSS more frequently asymptomatic and undergoing spontaneous closure during infancy ( $<2$ years old). Moreover, there are no reports of malignant liver tumors in IPSS (13)'(21). For these reasons, our study focuses on extrahepatic shunts only.

Because knowledge on the natural history and outcome of patients with Abernethy malformation are mainly based on paediatric series, there are no formal recommendations on the evaluation and management of adult patients with CEPS. Our study describes the clinical manifestations, natural history, current diagnostic procedures, treatments applied and outcome of a large cohort of patients with CEPS with the aim of proposing a guide to homogenously approach these patients.

This article is protected by copyright. All rights reserved. 


\section{Patients and Methods}

This is a multicenter observational retrospective international study performed in the setting of VALDIG Group (Vascular Liver Disease Interest Group) and the REHEVASC group (Registro Español de Enfermedades Vasculares Hepáticas).The 23 participating hospitals are listed in Annex 1. The local ethical committees of each hospital approved the study in accordance with the International Guideline for Ethical Review of Epidemiological Studies and principles of the Declaration of Helsinki.

\section{Patients and study design}

Every patient diagnosed in any of the participating hospitals was considered for inclusion if they had at least one imaging study demonstrating the presence of CEPS.

Demographic, clinical and imaging data at diagnosis were retrospectively recorded as well as follow-up data and the therapeutic approaches in a specifically designed case report form. HE, PaHT, HPS, HCC and adenoma were considered major CEPS complications.

\section{Results}

Since June 1989 until May 2018, 76 eligible patients were identified at the 23 participating centers. Ten of these patients were excluded from the study: 3 because CEPS diagnosis was not conclusive, 3 because they presented an intrahepatic portosystemic shunt instead of CEPS and 4 because they had concomitant cirrhosis at diagnosis making it difficult to establish whether symptoms were due to cirrhosis or to CEPS. Finally, 66 patients with Abernethy malformation were included. Of those, 10 patients had been previously reported as isolated case reports or as part of small series $(4,22-24)$. Follow-up of these patients has been updated for the current study. Out of the 66 patients, $35(53 \%)$ were men. At diagnosis, patients had a median age of 21 years (range: 0-66 years). The median follow up was of 5.2 years (range: $0-22$ ) and the median age at the end of follow-up was 30 years (range: 0-68 years). Table 1 shows baseline characteristics of patients at diagnosis and Supplementary Figure 1 shows the distribution of age at diagnosis. Table 2 summarizes the findings of the current study.

This article is protected by copyright. All rights reserved. 


\section{Diagnosis}

All patients were incidentally diagnosed through imaging tests. In 13 of the 66 patients $(19.7 \%)$, the imaging test was performed because patients already presented with symptoms potentially attributable to CEPS: HE in 6 cases and dyspnea in 7 cases. In the remaining 53 patients (80.3\%), the imaging study was performed for other different reasons (Table 1).

There were no significant differences in the age at diagnosis between patients with symptoms at diagnosis (22 years, range: $2-64$,) or without symptoms (24 years, range: $0-66)(p=0.57)$.

Imaging studies demonstrating the presence of CEPS were a Doppler ultrasound in 3 patients (4.5\%), CT scan in 36 patients (54.5\%), MRI in 10 patients (15.2\%) and both CT and MRI in 17 patients (25.8\%). Based on these imaging studies, type I CEPS was identified in 39 patients $(59 \%$, type la and type $\mathrm{lb}$ in 7 and 32 patients, respectively) and type II in the remaining 27 patients (41\%). There were no significant differences in sex, association to other congenital malformations or age at diagnosis between type I and II CEPS. Remarkably, 8 patients classified as type I CEPS at CT scan were shown to actually have intrahepatic portal vein inflow when further studied with an endovascular shunt occlusion test. This occlusion test was not performed in all patients, making it impossible to rule out that other patients previously considered as type I had indeed type II CEPS with residual hypoplastic portal branches.

Although not necessary for diagnosis, in 19 patients (29\%) a liver biopsy was performed. Liver biopsies were considered normal in 3 patients while the remaining 16 patients presented structural changes in different degrees: in 9 patients there were absent or hypoplastic portal vein branches in the portal tracts, combined in some cases with congestive sinusoids and large arterial branches; 8 patients presented low degree of periportal fibrosis and 3 patients presented moderate steatosis. Liver blood test at diagnosis showed a preserved liver function with a median albumin of $37.5 \mathrm{mg} / \mathrm{dL}$ (range $30-46 \mathrm{mg} / \mathrm{dL}$ ), prothrombin ratio of $80 \%$ (range: $60-100 \%$ ) and bilirubin of $1.1 \mathrm{mg} / \mathrm{dL}$ (range: $0.17-4 \mathrm{mg} / \mathrm{dL})$.

\section{Associated Congenital malformations}

Twenty-nine patients (44\%) presented one or multiple associated malformations (Supplementary table 1). Cardiac defects were the most common defects $(n=20)$, followed by musculoskeletal abnormalities $(\mathrm{n}=9)$.

Hepatic Encephalopathy

This article is protected by copyright. All rights reserved. 
Nineteen patients (29\%) developed hepatic encephalopathy (HE) at some time during the study. The median age at HE diagnosis was 12 years (range: 5-65). HE was already present at diagnosis in 14 patients while the other 5 patients developed HE during follow-up.

Different patterns of HE were observed with a clear preponderance of chronic HE: 14 patients had persistent HE with permanent cognitive impairment; 2 patients had recurrent HE and 3 patients had episodic HE. In most patients the episodes of HE were of moderate intensity (grade I in 11 patients and grade II in 7 according to West-Haven criteria) and only one patient had grade III-IV HE. Presence of minimal HE was tested only in 2 asymptomatic patients with the critical flicker frequency test (CFF) and psychometric hepatic encephalopathy score (PHES) tests and one of them was classified as having minimal $\mathrm{HE}$.

The cumulative probability of $\mathrm{HE}$ at 10,20 and 30 years was $13 \%, 24 \%$ and $28 \%$. (Figure 1a). Sixteen out of the 19 patients with HE (84\%) presented with HE before the age of 25 while the remaining 3 patients had HE after the age of 50 . These 3 patients represent $27 \%$ of all patients with an age above 50. There were no significant differences in the incidence of HE between patients with type I or type II CEPS (Figure 1b, $\mathrm{p}=0.57$ ). In addition, there were no significant associations between HE development and sex, presence of associated malformations, parameters assessing liver function or presence of other CEPS manifestations.

Brain MRI was performed in 19 of the 66 patients (29\%). In 10 of them MRI was performed after developing HE and in 9 cases in the absence of previous HE (Supplementary Table 2). Presence of high signal intensity in the globus pallidum (GP) on T1-weighted images was more frequent in patients with HE ( $70 \%$ vs. $30 \%, p=0.17)$. Among the 3 patients without HE but with GP high intensity at MRI, 1 of them developed HE 4 years later, while none of the 6 without neither HE nor GP high intensity presented clinical HE after a median follow-up of 20 years (range 1.5-35). No relation was detected between the grade or pattern of HE and the presence/absence of high GP intensity.

Venous serum ammonia levels determined in 20 patients at diagnosis were high in almost all of them (median $89.5 \mu \mathrm{mol} / \mathrm{L}$ range: $48-273$, normal value $<50 \mu \mathrm{mol} / \mathrm{L}$ ). Eight patients had already developed $\mathrm{HE}$ at the moment of the test, but no significant differences in serum ammonia levels were found with those of the remaining 12 patients who had not developed HE: $106 \mu \mathrm{mol} / \mathrm{L}$ (range: 48-273) for HE vs. $99 \mu \mathrm{mol} / \mathrm{L}$ (range: $57-163$ ) for no HE. Similarly, there was no relation between serum ammonia levels

This article is protected by copyright. All rights reserved. 
and other clinical characteristics of patients such as CEPS type, age at testing, sex or brain MRI findings.

Thirteen patients had additional serum ammonia tests during follow-up (after liver transplant in 1, shunt closure in 6 and after medical treatment of $\mathrm{HE}$ in 6), showing a significant reduction in the levels in all but one patient (median ammonia levels $51 \mu \mathrm{mol} / \mathrm{L}$, range 23-95).

\section{Liver Nodules}

All patients were screened for nodules. In 43 of the 66 patients (65\%) liver nodules were identified. Out of the 43 patients with nodules, 9 patients $(21 \%)$ had a single nodule, 24 patients (55\%) had 2 to 10 nodules, 5 patients (12\%) had 10 to 20 nodules and 5 patients had more than 20 .

The nature of liver nodules was established by biopsy in 25 patients $(58 \%)$ and through imaging characterization in the remaining 18 patients. Supplementary Table 3 shows liver nodules classification in patients with and without biopsy.

Overall, 18 patients had neoplastic lesions (8 HCC and 10 adenomas) and 25 patients had nonneoplastic nodules (nodular regenerative hyperplasia in 7, focal nodular hyperplasia in 12 and nonspecific in 6). HCC was diagnosed at a median age of 39 years (range: $32-53$ ) and had a median size of $94 \mathrm{~mm}$ (range: $11-160 \mathrm{~mm}$ ); 4 of them were identified at CEPS diagnosis while the other 4 were identified after a median of 9 years of follow-up (range 4-16). Adenomas were diagnosed at a median age of 18 years (range: $4-46$ ) and had a median size of $76 \mathrm{~mm}$ (range: 11-180). Benign nodules were identified at a median age of 20 years (range: 7-52) and had a median size of $36 \mathrm{~mm}$ (range: 10 $100 \mathrm{~mm})$.

AFP levels were evaluated in 39 patients, including all patients with HCC. It was found that AFP was elevated only in 5 patients, 4 of whom presented HCC (median $1459.5 \mathrm{U} / \mathrm{ml}$, range 74-7627). The remaining 4 patients with $\mathrm{HCC}$ had normal levels of AFP. The only patient with high AFP without HCC remained without evidence of $\mathrm{HCC}$ until the end of his follow-up 2 years later. All cases of HCC were identified in patients with type I CEPS (type I $n=8$, type $I I n=0 ; p=0.03$ ). Seven out of the 8 patients with HCC were male $(p=0.05)$, corresponding to a cumulative incidence of HCC of $40 \%$ in males with type I CEPS older than 30 years. Regarding adenomas, there were no significant differences in their distribution in relation to CEPS type (type I $n=7$, type $I \mathrm{n}=3 ; \mathrm{p}=0.5$ ). All 10 patients with adenoma were female (male $n=0$, female $n=10 ; p=0.001$ ). Thus, in this cohort, $33 \%$ of female patients developed adenomas.

This article is protected by copyright. All rights reserved. 
Twenty of the 25 patients with benign nodules had at least one imaging study during follow-up (median time from first to last imaging study: 9.8 years, range: 0.3-20years). In most cases, the size and number of nodules remained stable. However, a small increase in size was observed in 3 patients (median increase $13 \mathrm{~mm}$, range: 5-30 mm) and additional nodules appeared during follow-up in 4 patients.

Pulmonary arterial hypertension (PaHT) and hepatopulmonary syndrome (HPS)

Ten patients had dyspnea at a median age of 18 years (range: 2-42years). Pulmonary arterial hypertension was diagnosed in 8 of them (in 6 it was the main symptom leading to CEPS diagnosis, with a mean pulmonary arterial pressure of $46.5 \mathrm{mmHg} \pm 5.3$ ), and hepatopulmonary syndrome in the other 2 (severe in both of them, with hypoxemia $\mathrm{PaO}_{2}<50 \mathrm{mmHg}$ requiring domiciliary oxygen). Pulmonary arterial circulation was evaluated with echocardiography $(n=18)$ and/or Swan-Ganz right heart catheterization $(n=3)$ in 19 of $37(51 \%)$ asymptomatic patients, revealing PaHT in two of them (ages of 1 and 10 years, respectively). Sex, presence of other congenital malformations or cardiac defects, type of CEPS, development of HE or nodules was not associated with the presence of PaHT or HPS.

The cumulative incidence of at least one major CEPS complication (HE, PaHT, HPS, HCC and adenoma) at 20, 30 and 40 years was 35\%, 45\% and 58\%, respectively (Figure 2).

\section{Treatment and Outcome}

Three out of the 19 patients with HE presented subjective improvement with lactulose and rifaximin, while the other 16 presented persistent or recurrent HE despite receiving medical treatment. Four of the patients with persistent HE underwent shunt closure resulting in cognitive improvement and a decrease in ammonia blood level in all of them. One of them had a brain MRI 2 years after shunt closure showing a reduction in GP hyperintensity. Two of the patients with HE underwent liver transplantation (one because he presented HCC and the other adenoma), and they evolved with resolution of HE.

Seven of the 10 patients with PaHT remained stable with specific medical treatment (median followup 7.5 years, range 0-37). The other 3 patients underwent shunt closure, observing a clear clinical improvement in 2 patients (in one confirmed also by cardiopulmonary catheterization) and making it possible to stop medical treatment. The main differences between the 2 patients improving after shunt

This article is protected by copyright. All rights reserved. 
closure and the one in whom there was no discernible improvement were as follows: younger age (23 and 24 years vs 34 years), lower mean pulmonary pressure (44 and $49 \mathrm{mmHg}$ vs $51 \mathrm{mmHg}$ ) and shorter duration since diagnosis of PaHT ( 2 and 16 years vs 29 years) in those that improved.

Two patients with HPS underwent shunt closure, both at the age of 41 , which was followed by resolution of hypoxemia and dyspnea 1 month and 1 year after the procedure, respectively.

Among the 8 patients with $\mathrm{HCC}$, one patient presented an acute hemoperitoneum and died shortly afterwards. Six patients were submitted to surgical hepatectomy: 3 showed no HCC recurrence after a median follow-up of 36 months (range 4-48) after surgery, while the other 3 developed HCC recurrence after a median of 44 months (range: $38-48$ ), one of them eventually dying. One patient with concomitant HE underwent liver transplantation without further complications and was free of disease after 6 months of follow-up.

Out of 10 patients with adenomas, 3 were treated with surgical hepatectomy and had no complications after a median follow-up of 12 months (range: 3-84 months). Five were transplanted (one of them with concomitant $\mathrm{HE}$ ) and, among them, one died 5 months later due to septic complications. One patient underwent shunt closure but no changes in adenoma size $(120 \mathrm{~mm})$ were observed after 2 years of follow-up. An additional patient with a small adenoma $(11 \mathrm{~mm})$ was managed conservatively and remained stable after 4 years of follow-up.

In 5 asymptomatic patients shunt closure was performed as a preemptive measure at a median age of 20 years old (range: 5-31). No complications were registered until the end of their follow-up (median of 5.5 years after shunt closure, range: 1-12years). Interestingly, 4 of these 5 patients presented benign nodules and all of them experienced a decrease in their size and/or number.

Overall, 15 patients (23\%) underwent shunt closure, their main features according to the type of CEPS are summarized in Table 3. The main indication was chronic HE in 4 patients, PaHT in 3 patients, HPS in 2 patients, presence of a large adenoma in 1 patient and preemptive in 5 patients. Shunt closure was performed at a median age of 17.5 years (range: $3-41)$ using a surgical ligation in 11 patients and a percutaneous endovascular technique in the remaining 4 patients. The election of surgical vs endovascular approach was guided mainly by the size of the shunt. Eight patients had type I and the other 7 had type II CEPS. It is important to underline that in the 8 type I CEPS patients,

This article is protected by copyright. All rights reserved. 
the intrahepatic portal vein branches were identified when a shunt occlusion was performed, in contrast with the lack of connection between the portal venous axis and the intrahepatic circulation described by the conventional imaging studies.

In 6 patients the procedure was done in 2 steps to facilitate progressive redirection of portal blood flow to the liver. Remarkably, in one patient with type I CEPS the occlusion test showed a marked increase in portal pressure without adequate drainage, making a TIPS (transjugular intrahepatic portosystemic shunt) placement deemed to decompress the portal venous system before completing shunt closure (Supplementary Figure 2). Probably because of these safety precautions, only one of the 15 patients with shunt closure presented a thrombosis of the mesenteric and splenic vein, a complication directly attributable to the shunt closure procedure.. This patient was on anticoagulation during 1 year but no regression of the thrombus was achieved. In the other 14 patients there were neither portal hypertension related complications nor signs of bowel ischemia. None of the 15 patients developed new CEPS complications after a median of 2.5 years of follow-up after shunt closure (range: $0-12$ years). In 4 of the 15 patients a median increase of $428 \mathrm{~cm}^{3}$ (range 179-598) in liver volume was reported.

There were 21 patients (32\%) without major complications of CEPS that remained asymptomatic without being submitted to any intervention until the end of their follow-up at a median age of 33 years (range: 5-67).

\section{Discussion}

CEPS are an infrequent malformation characterized by the presence of a communication between the portal vein (or one of its afferent veins) and the inferior cava vein diverting the blood flow to the systemic circulation. These alterations in the splanchnic circulation produce hemodynamic and physiological changes that lead to severe complications. Nevertheless, knowledge about CEPS is scarce as the low incidence of this malformation has prevented the realization of large studies. In the current study, we have retrospectively enrolled 66 patients with congenital extrahepatic portosystemic shunts, thus presenting the largest series of patients with CEPS up to now with a long follow-up with more than $80 \%$ of patients achieving adulthood. This has allowed us to further characterize the manifestations and course of CEPS and to analyze current management strategies. The study points

This article is protected by copyright. All rights reserved. 
out the highly variable approaches among the different centers. Based both on our results and on previous reports, we suggest a work-up for CEPS in an attempt to homogenize its management.

The results of the current study show that patients with CEPS can remain asymptomatic until late in the disease and be undiagnosed until an advanced age. This is in contrast with previous case reports and series where approximately $75 \%$ of CEPS were diagnosed during childhood (3)(16) (19)(25)(26). However, because most of the participating centers take care of adult patients, a bias evaluating the age of our patients at diagnosis cannot be discarded.

In the current cohort, CEPS proved to be a severe and life threatening condition in many patients ( $68 \%$ of patients included in the study developed severe complications related to CEPS). It is important to underline that we may be underestimating the real incidence of CEPS complications as some of them can be present but asymptomatic and remain undiagnosed if not specifically screened for. Similarly, however, as data from a systematic population screening are not available, we also cannot estimate the burden of uncomplicated patients with CEPS that remain undiagnosed and that may never develop symptoms.

In agreement with previous small series (27), HE was the main complication in our cohort affecting more than one every four patients. This is highly relevant since even mild and minimal HE have proven to diminish quality of life. The present study was unable to identify any clinical or biochemical parameter (including ammonia levels) able to predict HE development. Out of the 47 patients without overt HE, minimal HE was only evaluated in 2 patients. Minimal HE could present in atypical forms and be difficult to detect if not suspected, especially in patients with CEPS that frequently present associated congenital malformations and intellectual disabilities. Thus, future studies systematically evaluating the presence of minimal HE are warranted.

It is possible, however, that the finding of high GP intensity in brain MRI in patients without HE could identify patients at a higher risk of developing HE or having minimal HE. Nevertheless, our data also show that the lack of high GP intensity in brain MRI cannot confidently exclude the presence of HE in equivocal cases because this alteration was absent in $20 \%$ of cases with clear overt HE. Unfortunately, the number of patients without HE studied with brain MRI was very small, precluding any definitive conclusion. It will be of great interest to include brain MRI in the work-up of newly diagnosed patients with CEPS.

This article is protected by copyright. All rights reserved. 
Our study also suggests that HE may be bimodal, with most patients developing HE before the age of 25 but with a second rise in new-onset HE after the age of 50 . Although we do not have an explanation for this finding, we can speculate that loss of brain reserve associated with aging may facilitate the revelation of HE (28).

Liver nodules have been previously described in as much as $40 \%$ of the reported patients with CEPS, being mainly focal nodular hyperplasia and regenerative nodular hyperplasia (70\%). Hepatocellular carcinoma (20\%) and adenomas (10\%) have been described in a lower proportion (16-19). In our study, the overall cumulative incidence of liver nodules was higher than previously reported (4)'(20)'(27), reaching $65 \%$ of patients. More importantly, a relevant and higher proportion of patients than previously described in the literature had adenomas and HCC, probably in the context of better imaging accuracy in the recent years and also in relation to the older age of our patients. In this regard, even though pediatric patients with CEPS have been described to develop HCC (29), in our cohort HCC was a late complication appearing after the age of 30.

Previous observations suggested that the development of liver nodules (regardless of their nature) is more frequent in type I CEPS than in type II CEPS (17)' (18). In our cohort this was the case only for HCC, which appeared almost exclusively in type I CEPS, probably in the setting of more severe alterations in liver perfusion and excessive arterialization due to the lack of portal blood flow (30)'. In our series HCCs were seen almost exclusively in men, while adenomas were observed only in women. This sex predilection is similar to that observed in general population and does not have a clear explanation. However, evidence suggests that HCC is an androgen sensitive tumor (31) and that the increased estrogen levels of females may enhance the risk of developing benign liver tumors (32). Nevertheless, it should be noted that adenomas have been also reported in males with CEPS in the literature (19). These data suggest the need of performing a careful and periodic screening for liver nodules in patients with CEPS and therefore it would seem sensible to adopt the cirrhosis strategy of HCC surveillance every six months.

Patients with CEPS have been reported to develop severe pulmonary complications such as $\mathrm{PaHT}$ (19)'(33). and, less frequently, hepatopulmonary syndrome (27)' (14). The development of HPS and $\mathrm{PaHT}$ could be in relation to intestinal vasoactive mediators $(14,15,34)$ that, having bypassed the liver and not being properly metabolized, reached the pulmonary vascular bed inducing a longstanding pulmonary vasoconstriction in the case of PAHT $(15,16)$ or, on the contrary, pulmonary

This article is protected by copyright. All rights reserved. 
vasodilation in the case of HPS. Our study confirms that $\mathrm{PaHT}$ is a significant problem in patients with CEPS causing symptomatic dyspnea in $80 \%$ of cases. Moreover, in this cohort $11 \%$ of asymptomatic patients also presented PaHT when specifically screened for it. Because PaHT was identified at a wide range of age, screening for PaHT may be worthy to be repeated during follow-up. Because performing periodic cardiac catheterization can be inconvenient, it seems reasonable to recommend using echocardiogram for follow-up PaHT screening. The study should be subsequently completed with cardiopulmonary catheterization if indirect signs of PaHT were found. Hepatopulmonary syndrome appears to be less frequent than PaHT, with prevalence in our study of $3 \%$. However, as mild forms of HPS might be asymptomatic and an exhaustive pulmonary study was not performed in all the patients of our cohort, its real prevalence cannot be estimated. It would be advisable to perform a baseline exhaustive screening of pulmonary complications and, afterwards, periodic screening with transcutaneous oxygen saturation measurements could be helpful for early detection of its appearance during follow-up.

Fifteen patients $(23 \%)$ were submitted to shunt closure, at a median age of 17 years, but as young as at 3 years and as late as at 41 years. It is important to remark that shunt closure restoring portal blood flow to the liver was possible even in patients with type I CEPS. This reinforces the theory that the assessment of intrahepatic portal veins patency based only in CT-Scan or MRI without performing an occlusion test might be misleading because residual hypoplastic veins might be only recognized through balloon occlusion venography, as showed by several previous reports (3)'(4)(19)'(27)'(35)'(36). The current study confirms this relevant finding given that in all type I CEPS patients that underwent an occlusion test, remnants of the intrahepatic portal vein branches were identified. Establishing confidently the presence or absence of intrahepatic veins is highly relevant when evaluating possible therapeutic options, as classically type I patients could only be treated with liver transplantation. Nevertheless, the type I and II CEPS classification is still useful because, even if the intrahepatic portal vein branches may be also present in type I CEPS, they are hypoplastic and leading less, if any, flow to the liver (3)', which could have pathophysiological consequences like more frequent HCC development as we have seen in our cohort.

This article is protected by copyright. All rights reserved. 
Shunt closure was always performed after checking that the procedure was associated with an adequate redirection of portal blood flow to the liver, usually requiring a two-step intervention promoting the stenosis of the portal-collateral circulation with the ensuing increase in the portal system pressure leading to the reopening of the hypoplastic portal branches. Some authors have suggested that prophylactic anticoagulation could also be useful to prevent thrombosis after shunt closure $(19,20)$. Our results coincide with previous reports $(5)^{\prime}(16)$ suggesting that shunt closure has a huge efficacy managing most CEPS complications and, most interestingly, preventing its appearance. Therefore, shunt closure must be always considered in symptomatic patients and should also be considered as a prophylactic treatment early in the evolution of the disease to prevent the development of severe complications. Nevertheless, it cannot be assured that the risk of complications completely disappears with the occlusion of the shunt, and thus HCC surveillance should still be performed until more data are available.

In the current cohort, shunt closure was especially useful in improving HE but was less effective in patients with long-standing and severe PaHT, probably because the highest the pressure, the less improvement after closure can be expected. Although the only patient with adenoma in our cohort did not improve after shunt closure, partial adenoma regression after shunt closure has been reported (19).

The present study shows that the current diagnostic and therapeutic approach of CEPS is highly variable depending on each center policy. We consider that consensus guidelines would improve the knowledge of the disease and the management of these patients. Thus, based on the results of our cooperative study, in Figure 3 we propose a management algorithm for all patients with CEPS.

\section{Conclusion}

This is the largest CEPS cohort until now and the only one in which most patients have reached adulthood at the end of follow-up, which could explain some of the clinical differences found in comparison with previous reports. CEPS patients may develop significant morbidity and lifethreatening complications. Therefore, screening for asymptomatic complications and close surveillance is needed. Shunt closure should be considered both in a therapeutic and prophylactic approach.

This article is protected by copyright. All rights reserved. 


\section{Bibliography}

1. Glyn Morgan RS. Congenital absence of the portal vein: Two cases and a proposed classification system for portasystemic vascular anomalies. J. Pediatr. Surg. [Internet]. 1994;29:1239-1241. Available from: doi:10.1016/0022-3468(94)90812-5

2. Howard ER, Davenport M. Congenital extrahepatic portocaval shunts - The Abernethy malformation. J. Pediatr. Surg. 1997;32:494-497.

3. Lautz TB, Tantemsapya N, Rowell E, Superina RA. Management and classification of type II congenital portosystemic shunts. J. Pediatr. Surg. [Internet]. 2011;46:308-314. Available from: http://dx.doi.org/10.1016/j.jpedsurg.2010.11.009

4. Blanc T, Guerin F, Franchi-Abella S, Jacquemin E, Pariente D, Soubrane O, et al. Congenital Portosystemic Shunts in Children: a new anatomical classification correlated with surgical strategy. Ann Surg. 2014;260:188-198.

5. Franchi-Abella S, Gonzales E, Ackermann O, Branchereau S, Pariente D, Guérin F, et al. Congenital portosystemic shunts: diagnosis and treatment. Abdom. Radiol. (New York) [Internet]. 2018;43:2023-2036. Available from: http://www.ncbi.nlm.nih.gov/pubmed/29730740

6. Kanazawa H, Nosaka S, Miyazaki O, Sakamoto S, Fukuda A, Shigeta T, et al. The classification based on intrahepatic portal system for congenital portosystemic shunts. J. Pediatr. Surg. [Internet]. 2015;50:688-95. Available from: http://www.ncbi.nlm.nih.gov/pubmed/25840084

7. Witters P, Maleux G, George C, Delcroix M, Hoffman I, Gewillig M, et al. Congenital venovenous malformations of the liver: Widely variable clinical presentations. J. Gastroenterol. Hepatol. 2008;23.

8. Konstas AA, Digumarthy SR, Avery LL, Wallace KL, Lisovsky M, Misdraji J, et al. Congenital portosystemic shunts: Imaging findings and clinical presentations in 11 patients. Eur. J. Radiol. $2011 ; 80: 175-181$.

9. Stringer MD. The clinical anatomy of congenital portosystemic venous shunts. Clin. Anat. $2008 ; 21: 147-157$

This article is protected by copyright. All rights reserved. 
10. Gitzelmann R, Forster I, Willi U V. Hypergalactosaemia in a newborn: self-limiting intrahepatic portosystemic venous shunt. Eur. J. Pediatr. [Internet]. 1997;156:719-22. Available from: http://www.ncbi.nlm.nih.gov/pubmed/9296538

11. Ono H, Mawatari H, Mizoguchi N, Eguchi T, Sakura N. Clinical features and outcome of eight infants with intrahepatic porto-venous shunts detected in neonatal screening for galactosaemia. Acta Paediatr. [Internet]. 1998;87:631-4. Available from: http://www.ncbi.nlm.nih.gov/pubmed/9686654

12. Akahoshi T, Nishizaki T, Wakasugi K, Mastuzaka T, Kume K, Yamamoto I, et al. Portalsystemic encephalopathy due to a congenital extrahepatic portosystemic shunt: three cases and literature review. Hepatogastroenterology. [Internet]. 47:1113-6. Available from: http://www.ncbi.nlm.nih.gov/pubmed/11020891

13. Uchino T, Matsuda I, Endo F. The long-term prognosis of congenital portosystemic venous shunt. J. Pediatr. 1999;135:254-256.

14. Fu L, Wang Q, Wu J, Guo Y, Huang M, Liu T, et al. Congenital extrahepatic portosystemic shunt: an underdiagnosed but treatable cause of hepatopulmonary syndrome. Eur. J. Pediatr. 2016;175:195-201.

15. Yi J-E, Jung H-O, Youn H-J, Choi JY, Chun HJ, Lee JY. A case of pulmonary arterial hypertension associated with congenital extrahepatic portocaval shunt. J. Korean Med. Sci. 2014;29:604-608.

16. Sokollik C, Bandsma RHJ, Gana JC, van den Heuvel M, Ling SC. Congenital Portosystemic Shunt: characterization of a multisystem disease. J. Pediatr. Gastroenterol. Nutr. 2013;56:675-681.

17. Sharma R, Suddle A, Quaglia A, Peddu P, Karani J, Satyadas T, et al. Congenital extrahepatic portosystemic shunt complicated by the development of hepatocellular carcinoma. Hepatobiliary Pancreat. Dis. Int. 2015;14:552-557.

18. Sorkin T, Strautnieks S, Foskett P, Peddu P, Thompson RJ, Heaton N, et al. Case Report: Multiple beta-catenin mutations in hepatocellular lesions arising in Abernethy Malformation. Hum. Pathol. [Internet]. 2016;53:153-158. Available from:

This article is protected by copyright. All rights reserved. 
http://www.sciencedirect.com/science/article/pii/S0046817716300089

19. Franchi-Abella S, Branchereau S, Lambert V, Fabre M, Steimberg C, Losay J, et al. Complications of congenital portosystemic shunts in children: therapeutic options and outcomes. J. Pediatr. Gastroenterol. Nutr. 2010;51:322-330.

20. Chocarro G, Virginia M, Jose A, Encinas L, Vilanova A, Hernandez F, et al. Congenital Portosystemic Shunts : Clinic Heterogeneity Requires an Individual Management of the Patient. Eur J Pediatr Surg. 2016;26:74-80.

21. Francois B, Gottrand F, Lachaux A, Boyer C, Benoit B, De Smet S. Outcome of intrahepatic portosystemic shunt diagnosed prenatally. Eur. J. Pediatr. [Internet]. 2017;176:1613-1618. Available from: http://www.ncbi.nlm.nih.gov/pubmed/28913555

22. Vicente N, Pérez M, Gander R, Segarra A, Leganés C, Bueno J. [Congenital portosistemic shunt. Importance of early treatment]. Cir. Pediatr. [Internet]. 2015;28:67-73. Available from: http://www.ncbi.nlm.nih.gov/pubmed/27775284

23. Nacif LS, Paranaguá-Vezozzo DC, Galvão FHF, Rocha MS, Andraus W, Carrilho FJ, et al. Significance of CT scan and color Doppler duplex ultrasound in the assessment of Abernethy malformation. BMC Med. Imaging [Internet]. 2015;15:37. Available from: http://www.biomedcentral.com/1471-2342/15/37

24. Witjes CDM, ljzermans JNM, Noordegraaf AV, Tran TCK. Management strategy after diagnosis of Abernethy malformation: a case report. J. Med. Case Rep. [Internet]. 2012;6:167. Available from: https://jmedicalcasereports.biomedcentral.com/articles/10.1186/1752-1947-6167

25. Gong Y, Zhu H, Chen J, Chen Q, Ji M, Pa M, et al. Congenital portosystemic shunts with and without gastrointestinal bleeding - case series. Pediatr. Radiol. 2015;45:1964-1971.

26. Ikeda S, Sera Y, Ohshiro H, Uchino S, Uchino T, Endo F. Surgical indications for patients with hyperammonemia. J. Pediatr. Surg. 1999;34:1012-1015.

27. Guérin F, Blanc T, Gauthier F, Abella SF, Branchereau S. Congenital portosystemic vascular malformations. Semin. Pediatr. Surg. [Internet]. 2012;21:233-244. Available from: http://dx.doi.org/10.1053/j.sempedsurg.2012.05.006

This article is protected by copyright. All rights reserved. 
28. Amodio P, Montagnese S, Spinelli G, Schiff S, Mapelli D. Cognitive reserve is a resilience factor for cognitive dysfunction in hepatic encephalopathy. Metab. Brain Dis. [Internet]. 2017;32:1287-1293. Available from: http://www.ncbi.nlm.nih.gov/pubmed/28573602

29. Benedict M, Rodriguez-Davalos M, Emre S, Walther Z, Raffaella Morotti. Congenital Extrahepatic Portosystemic Shunt (Abernethy Malformation Type lb) with Associated 2 Hepatocellular Carcinoma: Case report and Literature Review. Pediatr. Dev. Pathol. [Internet]. 2016;Available from: doi: 10.2350/16-01-1761-CR.1

30. Gülşen Z, Yiğit H, Demir P. Multiple regenerative nodular hyperplasia in the left infrarenal vena cava accompanied by abernethy malformation. Surg. Radiol. Anat. [Internet]. 2016;38:373378. Available from: http://link.springer.com/10.1007/s00276-015-1460-5

31. Naugler WE, Sakurai T, Kim S, Maeda S, Kim K, Elsharkawy AM, et al. Gender disparity in liver cancer due to sex differences in MyD88-dependent IL-6 production. Science [Internet]. 2007;317:121-4. Available from: http://www.ncbi.nlm.nih.gov/pubmed/17615358

32. Baek S, Sloane CE, Futterman SC. Benign liver cell adenoma associated with use of oral contraceptive agents. Ann. Surg. [Internet]. 1976;183:239-42. Available from: http://www.ncbi.nlm.nih.gov/pubmed/176959

33. Ohno T, Muneuchi J, Ihara K, Yuge T, Kanaya Y, Yamaki S, et al. Pulmonary hypertension in patients with congenital portosystemic venous shunt: a previously unrecognized association. Pediatrics [Internet]. 2008;121:e892-9. Available from: http://www.ncbi.nlm.nih.gov/pubmed/18362102

34. Morikawa N, Honna T, Kuroda T, Kitano Y, Fuchimoto Y, Kawashima N, et al. Resolution of hepatopulmonary syndrome after ligation of a portosystemic shunt in a pediatric patient with an Abernethy malformation. J. Pediatr. Surg. 2008;43.

35. Murray CP, Yoo SJ, Babyn PS. Congenital extrahepatic portosystemic shunts. Pediatr. Radiol. 2003;33:614-620.

36. Yonemitsu H, Mori H, Kimura T, Kagawa K, Tsuda T, Yamada Y, et al. Congenital extrahepatic portocaval shunt associated with hepatic hyperplastic nodules in a patient with Dubin-Johnson syndrome. Abdom. Imaging [Internet]. 25:572-5. Available from:

http://www.ncbi.nlm.nih.gov/pubmed/11029086

This article is protected by copyright. All rights reserved. 


\begin{tabular}{|c|c|c|c|}
\hline \multicolumn{2}{|c|}{ Table 1. Baseline characteristics ( $n=66$ ) } & $\mathbf{N}$ & $\%$ \\
\hline \multicolumn{2}{|l|}{ Sex (male) } & 35 & 53 \\
\hline \multicolumn{4}{|l|}{ Reason Diagnosis } \\
\hline \multirow[t]{3}{*}{ CEPS Symptoms: } & & 13 & 19.7 \\
\hline & $\mathrm{HE}$ & 6 & 9.1 \\
\hline & Dyspnea & 7 & 10.6 \\
\hline \multicolumn{4}{|c|}{ Imaging study for: } \\
\hline & Abnormal liver test & 16 & 24.2 \\
\hline & Abdominal pain & 10 & 15.2 \\
\hline & Other malformations & 7 & 10.6 \\
\hline & Prenatal US & 2 & 3 \\
\hline & Others & 18 & 27.3 \\
\hline \multirow[t]{3}{*}{ Type I CEPS } & & 39 & 59 \\
\hline & Type la & 7 & \\
\hline & Type Ib & 32 & \\
\hline \multirow[t]{2}{*}{ Type II CEPS } & & 27 & 41 \\
\hline & & Median & Range, (SD) \\
\hline Age at diagnosis & & 21 & $0-66,(16.7)$ \\
\hline ALT U/L & & 36 & $10-103,(21.1)$ \\
\hline AST U/L & & 38 & $11-93,(17.9)$ \\
\hline GGT U/L & & 79 & $15-370,(95.1)$ \\
\hline Bilirubin $\mathrm{mg} / \mathrm{dL}$ & & 1.1 & $0.1-4,(0.94)$ \\
\hline Ammonia $\mu \mathrm{mol} / \mathrm{L}^{*}$ & & 101 & $48-273,(49.7)$ \\
\hline Prothrombin ratio & & 80 & $60-100,(13)$ \\
\hline
\end{tabular}

* Ammonia levels were determined in 20 patients

This article is protected by copyright. All rights reserved. 


\begin{tabular}{|l|l|l|l|l|}
\hline & $\begin{array}{l}\text { Age at } \\
\text { complication } \\
\text { (median, range) }\end{array}$ & $\begin{array}{l}\text { Sex } \\
\text { (male) }\end{array}$ & Type I CEPS & Shunt closure \\
\hline HE: $\mathbf{n = 1 9 ~ ( 2 9 \% ) ~}$ & $12(5-65)$ & $9(47 \%)$ & $12(63 \%)$ & 4 \\
\hline PaHT: $\mathbf{n = 1 0 ~ ( 1 5 \% ) ~}$ & $20(2-42)$ & $6(60 \%)$ & $6(60 \%)$ & 3 \\
\hline HPS: $\mathbf{n = 2 ~ ( 3 \% ) ~}$ & $41(41-41)$ & $1(50 \%)$ & 0 & 2 \\
\hline HCC: $\mathbf{n = 8 ( 1 2 \% )}$ & $39(32-53)$ & $7(87 \%)$ & $8(100 \%)$ & 0 \\
\hline Adenoma: (n= 10, 15\%) & $18(4-46)$ & 0 & $7(70 \%)$ & 1 \\
\hline $\begin{array}{l}\text { No main complications } \\
\text { (n= 21, 32\%) }\end{array}$ & $30(0-67)^{*}$ & $16(76 \%)$ & $13(62 \%)$ & 5 \\
\hline
\end{tabular}

*age at last follow-up

\begin{tabular}{|c|c|c|c|c|c|}
\hline \multirow{2}{*}{\multicolumn{2}{|c|}{ Shunt closure }} & \multicolumn{2}{|c|}{ Type I CEPS } & \multicolumn{2}{|c|}{ Type II CEPS } \\
\hline & & \multicolumn{2}{|c|}{8} & \multicolumn{2}{|c|}{7} \\
\hline \multirow[t]{2}{*}{ Technique } & Surgical & \multicolumn{2}{|l|}{5} & \multicolumn{2}{|l|}{6} \\
\hline & Endovascular & \multicolumn{2}{|l|}{3} & \multicolumn{2}{|l|}{1} \\
\hline \multirow[t]{2}{*}{ Steps } & One step & \multicolumn{2}{|l|}{5} & \multicolumn{2}{|l|}{4} \\
\hline & Two steps & \multicolumn{2}{|l|}{3} & \multicolumn{2}{|l|}{3} \\
\hline \multirow[t]{5}{*}{ Indication } & PaHT & 2 & Resolution in 1 & 1 & Resolution \\
\hline & HPS & \multicolumn{2}{|l|}{0} & 2 & Resolution \\
\hline & $\mathrm{HE}$ & 4 & Improvement in all & \multicolumn{2}{|l|}{0} \\
\hline & Adenoma & 1 & No changes & \multicolumn{2}{|l|}{0} \\
\hline & Prophylactic & 1 & No complications & 4 & No complications \\
\hline \multicolumn{2}{|c|}{ Complications } & \multicolumn{2}{|l|}{1} & \multicolumn{2}{|l|}{0} \\
\hline
\end{tabular}

This article is protected by copyright. All rights reserved. 
Figure 1a. Cumulative incidence of HE

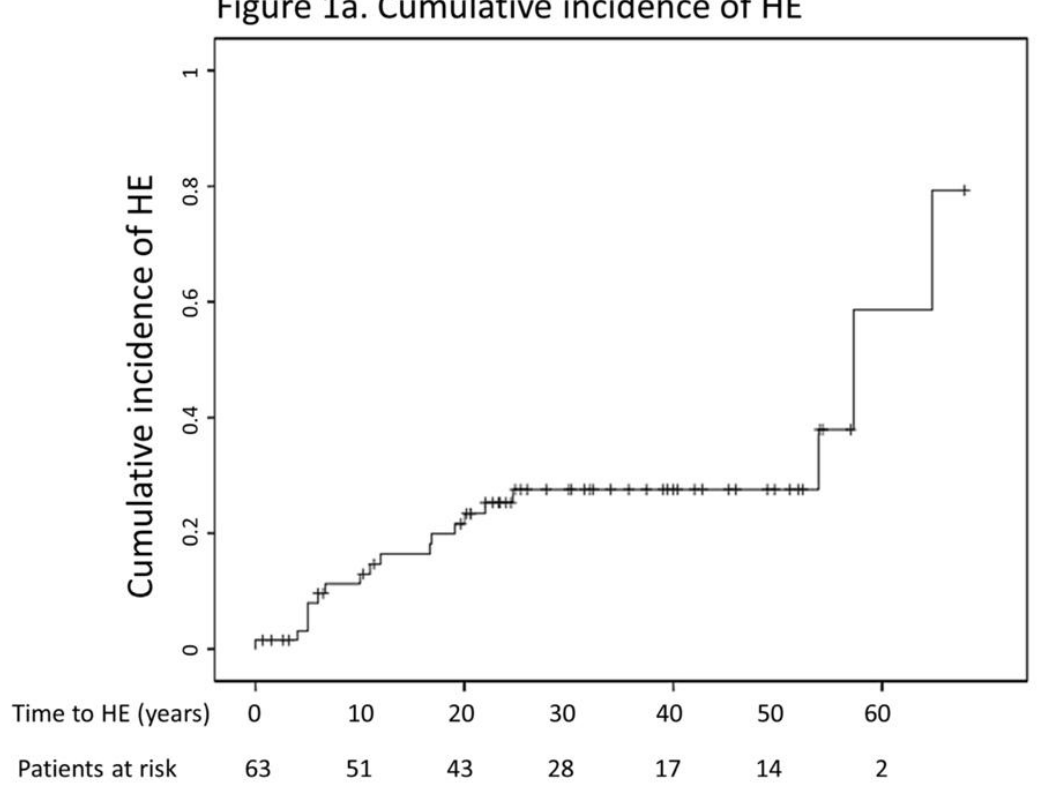

Figure 1b. Cumulative incidence of HE depending on CEPS Type

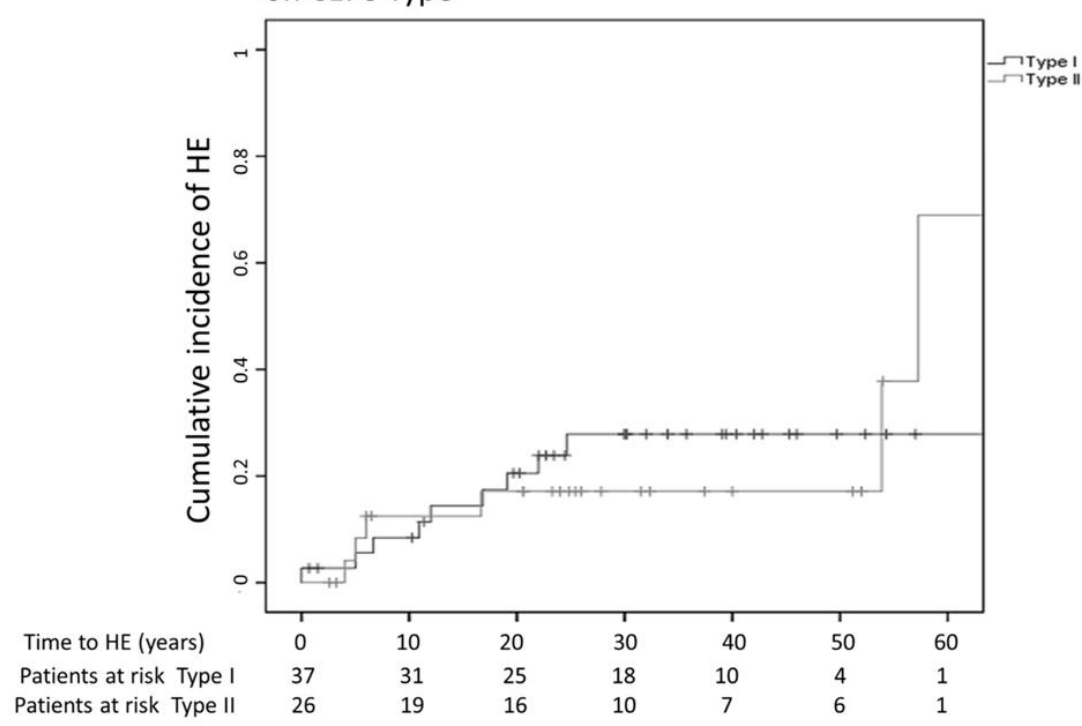

This article is protected by copyright. All rights reserved. 
Figure 2

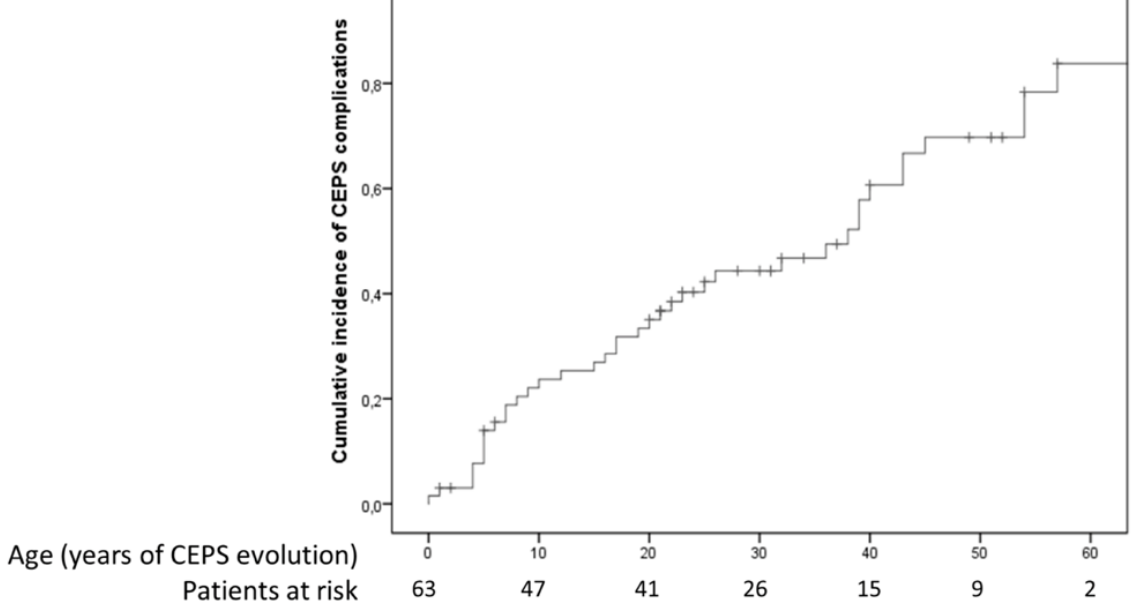

Figure

3

Abernethy Malformation suspicion

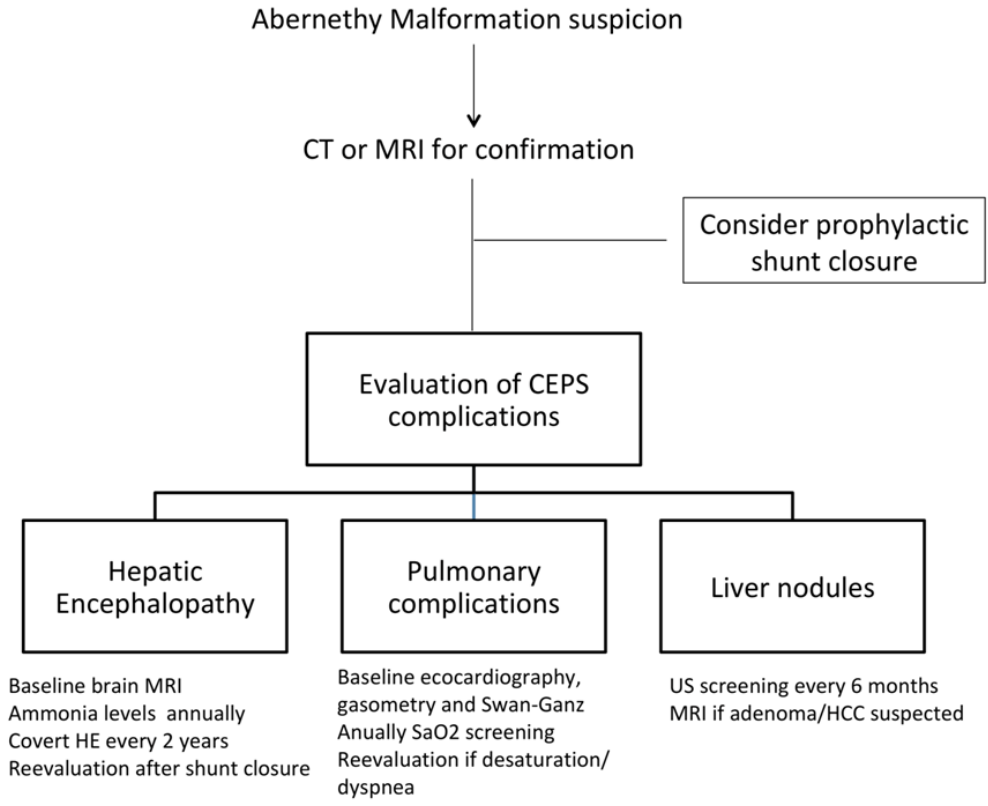

This article is protected by copyright. All rights reserved. 